\title{
TAXONOMY OF NATURE INSPIRED COMPUTATIONAL INTELLIGENCE IN DIGITAL IMAGE PROCESSING FOR HARSH WEATHER
}

\author{
Meenu \\ Research Scholar, \\ Mewar University, Chittorgadh, \\ Rajasthan, India
}

\author{
V.K.Panchal \\ Founder President \\ Computational Intelligence Research Group
}

\author{
Gourav kumar \\ Department of Software System \\ Birla Institute of Engineering and Technology , Pilani
}

\begin{abstract}
The concept of harsh weather image processing is generally vague, ambiguous and imprecise. Limited work is carried out in many outdoor computer vision applications. Recently the nature inspired Computational Intelligence technique has emerged as an efficient mechanism to handle diverse uncertainty characteristics. This paper presents the human mind based Computational Intelligence technique emerging in harsh weather vision enhancement. Researchers have shown keen Interest on the application of natural inspired techniques and proved as an efficient approach to build versatile and adaptable system to solve non linear problem which are much effective to use in the computer vision.
\end{abstract}

Keywords: Nature Inspired Computing, Computational Intelligence, Harsh weather, Haze effect, Satellite Images.

\section{INTRODUCTION}

In this modern era, image transmission and processing plays a crucial and prominent role. It would not be possible to retrieve information from satellite and medical images without the help of images processing techniques.

Recently, the nature inspired Computational Intelligence (CI) techniques which are inspired from the concept of artificial immune system, Swarm Intelligence based Techniques have immerged as an efficient mechanism to handle diverse uncertainty characteristics. Implementation of nature inspired computing has been vital with machine intelligence which behaves similar to human behavior and reasoning capability.

Researchers have shown keen interest on the application of nature Inspired computing in divergent domains. The areas and with techniques on which researchers have drawn the attention are here redefines and extends the taxonomy of nature Inspired computational Intelligence (CI). We have proposed the taxonomy of the most recent nature Inspired CI techniques that have been adapted till date for harsh weather future extraction and have also analyzed their performance. Here, Harsh weather image means the images of outdoor scenes which are usually degraded by the turbid medium (e.g. Particles and weather droplets) in the atmosphere [1][2].

Haze, fog and smoke are such phenomena due to atmospheric absorption and scattering. The irradiance received by the camera from the scene point is attenuated along the line of sight.

Furthermore, the incoming light is blended with the air light. The degraded images by harsh weather lose contrast and color fidelity as shown in figure 1 . Hence the amount of scattering depends on the distance of scene points from the camera.

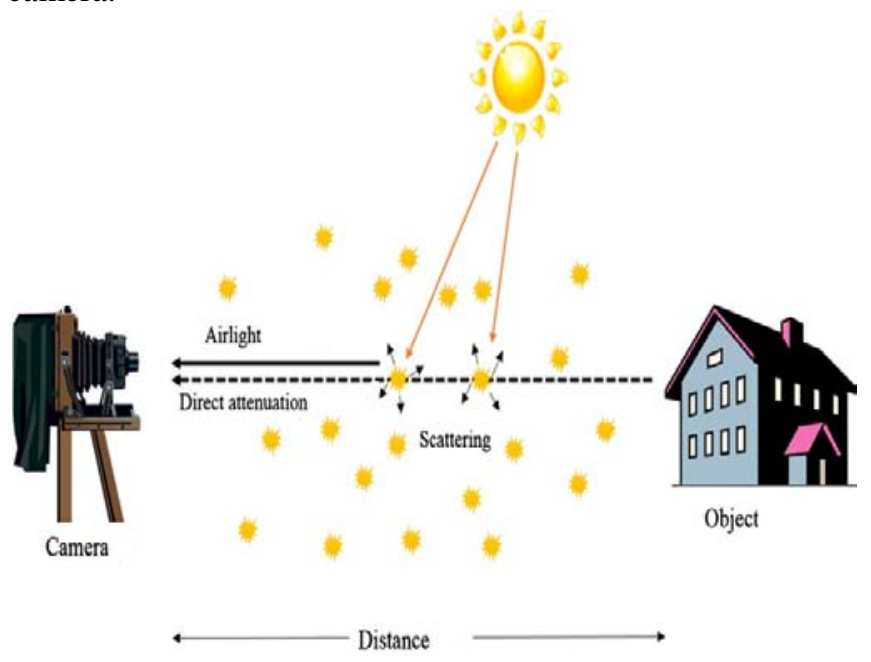

Figure 1: Scattering process of degraded image captured at camera.

The discussion in this paper is detailed explanation of various applications of image processing in Nature Inspired CI techniques and how these new algorithms find their way in the field of image processing. This paper is divided into four sections. Section II gives review of techniques for harsh weather. Section III gives brief Taxonomy on nature inspired CI Techniques. Section IV summarizes the proposed taxonomy of most recent nature inspired CI techniques that have been adapted till date for harsh weather feature extraction. Section V concludes and illustrates the future direction of this paper. 


\section{A REVIEW OF HARSH WEATHER TECHNIQUES USED}

This section explores the widely used methods /Techniques/Filters for enhancing the image degraded by the harsh weather.

The main pollutant of the atmosphere is particles and particle size is one of the most important characters of particle which affects all of other physical and chemical characters. The fine particles have extreme effect to human health and air visibility. Bad weather is the result of natural atmospheric changes. Poor visibility is considered as the effect of Particles size, concentration, and distribution and categorized due to bad weather. Poor visibility conditions are mainly categorized according to the bad weather conditions, types and sizes of the atmospheric particles. They are broadly classified into steady (fog, mist and haze) and dynamic (rain, snow) weather conditions, based on visual effects. Weather and their visibility conditions are related with particle types and sizes [1] which are described in table 1.

\section{Table 1. Weather conditions and associated particle types, sizes and concentrations (adapted from McCartney (1975).}

\begin{tabular}{|c|c|c|c|c|}
\hline $\begin{array}{c}\text { Weather } \\
\text { Conditio } \\
\text { n } \\
\end{array}$ & $\begin{array}{c}\text { Visibilit } \\
y\end{array}$ & $\begin{array}{c}\text { Particle } \\
\text { Type }\end{array}$ & $\begin{array}{l}\text { Radiu } \\
\text { s }(\mu \mathrm{m})\end{array}$ & $\begin{array}{c}\text { Concentratio } \\
\mathbf{n}\left(\mathrm{cm}^{-3}\right)\end{array}$ \\
\hline Air & Normal & $\begin{array}{l}\text { Molecul } \\
\mathrm{e}\end{array}$ & $10^{-4}$ & $10^{19}$ \\
\hline Haze & \multirow[t]{4}{*}{ Poor } & Aerosol & $\begin{array}{l}10^{-2}- \\
1\end{array}$ & $10^{3}-10$ \\
\hline Fog & & $\begin{array}{l}\text { Water } \\
\text { droplet }\end{array}$ & $1-10$ & $100-10$ \\
\hline Cloud & & $\begin{array}{l}\text { Water } \\
\text { droplet }\end{array}$ & $1-10$ & $300-10$ \\
\hline Rain & & $\begin{array}{l}\text { Water } \\
\text { drop }\end{array}$ & $\begin{array}{l}10^{2}- \\
10^{4}\end{array}$ & $10^{-2}-10^{-5}$ \\
\hline
\end{tabular}

Haze consists of sufficient smoke, dust, moisture and vapors suspended in air to impair visibility. The particles produced by those sources respond quickly of small water droplets when the humidity is high. Haze particle are larger than air molecules but smaller than fog droplets.

Fog evolves when the relative humidity of an air parcel approaches a saturated level. Then, some of the nuclei are grown by the condensation of water droplets. Hence fog and haze have similar origin and an increase in humidity is sufficient to turn haze into fog. This transition is quite gradual and an intermediate state is referred to a mist. While perceptible haze extended to an altitude of several miles. Fog is typically just a few hundred feet thick. A practical distribution between fog and haze lies in the greatly reduced visibility included by the former.

Cloud views differ from fog only at existing higher altitudes rather than sitting at ground level. While most clouds are made of water droplets like fog, some are composed of long ice crystals and ice coated dust grains. Details on the physics of clouds and precipitation can be found in Mason (1975). For Now, clouds are of less relevance to us as we restrict ourselves to vision at ground level rather than at high altitudes.

Rain and snow shows the process by which cloud droplets turn to rain is a complex one (Mason, 1975). When viewed up close, rain causes random spatial and temporal variations in images and hence must be dealt with differently from the more static weather conditions.

Most of existing methods in the field of computer vision are based on the hypothesis/assumption that the weather in outdoor images or videos is clear. However, different weather conditions such as rain, snow or haze will cause complex visual effects in images or videos, as shown in Figure 2. Zheng Zhang et al [3] The areas which have drawn the attention are weather condition / Image classification / Haze , Fog Free images / feature extraction / Air light estimation in which hitherto is looked for its solution in classical artificial Intelligence based on nature inspired technique of computational Intelligence .

Table 2. An overview of techniques used for harsh weather image enhancement.

\begin{tabular}{|c|c|c|c|c|}
\hline $\begin{array}{l}\text { S. } \\
\text { no }\end{array}$ & $\begin{array}{c}\text { Metho } \\
\text { d }\end{array}$ & $\begin{array}{c}\text { Inven } \\
\text { tor }\end{array}$ & Result & $\begin{array}{l}\text { ye } \\
\text { ar }\end{array}$ \\
\hline 1 & $\begin{array}{c}\text { Polari } \\
\text { zation } \\
\text { Metho } \\
\text { d }\end{array}$ & $\begin{array}{l}\text { Y.Y. } \\
\text { Sche } \\
\text { chner }\end{array}$ & $\begin{array}{l}\text { Polarization based methods } \\
\text { remove the haze effect through } \\
\text { two or more images taken with } \\
\text { different degrees of } \\
\text { polarization.[4] }\end{array}$ & $\begin{array}{l}20 \\
01\end{array}$ \\
\hline 2 & $\begin{array}{l}\text { Contra } \\
\text { st } \\
\text { based } \\
\text { metho } \\
\text { d }\end{array}$ & $\begin{array}{l}\text { Robb } \\
\text { y T. } \\
\text { Tan }\end{array}$ & $\begin{array}{l}\text { Identify that a haze-free } \\
\text { image must have higher } \\
\text { contrast compared with the } \\
\text { input hazy image and he } \\
\text { removes haze by maximizing } \\
\text { the local contrast of the } \\
\text { restored image[5]. }\end{array}$ & $\begin{array}{l}20 \\
08\end{array}$ \\
\hline 3 & $\begin{array}{l}\text { Dehaz } \\
\text { ing } \\
\text { Using } \\
\text { Color- } \\
\text { Lines }\end{array}$ & $\begin{array}{l}\text { R. } \\
\text { Fattal }\end{array}$ & $\begin{array}{l}\text { Estimates the albedo of the } \\
\text { scene and the medium } \\
\text { transmission under the } \\
\text { assumption that the } \\
\text { transmission and the surface } \\
\text { shading are locally } \\
\text { uncorrelated. This approach is } \\
\text { physically sound and can } \\
\text { produce impressive results in } \\
\text { fog and hazed images [6]. }\end{array}$ & $\begin{array}{l}20 \\
08\end{array}$ \\
\hline 4 & $\begin{array}{l}\text { CLAH } \\
\text { E } \\
\text { MET } \\
\text { HOD }\end{array}$ & $\begin{array}{l}\mathrm{Xu}, \\
\text { Zhiyu } \\
\text { an }\end{array}$ & $\begin{array}{l}\text { This method establishes a } \\
\text { maximum value to clip the } \\
\text { histogram and redistributes the } \\
\text { clipped pixels equally to each } \\
\text { gray-level. It can limit the } \\
\text { noise while enhancing the } \\
\text { image contrast. get effective } \\
\text { result on color image } \\
\text { degraded by fog and apply the } \\
\text { edge detection to the image[8]. }\end{array}$ & $\begin{array}{l}20 \\
09\end{array}$ \\
\hline
\end{tabular}




\begin{tabular}{|l|l|l|l|l|}
\hline & & & & \\
\hline 5 & $\begin{array}{l}\text { Dark } \\
\text { Chan } \\
\text { nel } \\
\text { Prior }\end{array}$ & $\begin{array}{l}\text { Kaim } \\
\text { ing } \\
\text { He }\end{array}$ & $\begin{array}{l}\text { Dark channel prior is used for } \\
\text { the estimation of atmospheric } \\
\text { light in the dehazed image to } \\
\text { get the more proper result. } \\
\text { This technique is mostly used } \\
\text { for non-sky patches, as at least } \\
\text { one color channel has very low } \\
\text { intensity at some pixels[7]. }\end{array}$ & 11 \\
\hline
\end{tabular}
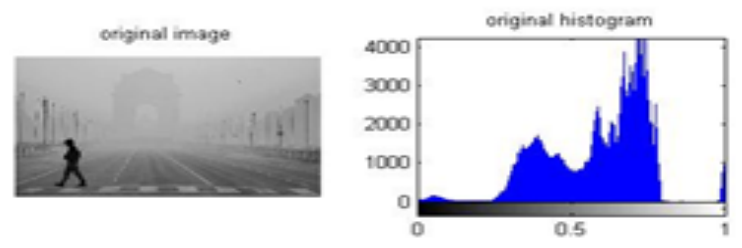

(a) Fogy Image with histogram
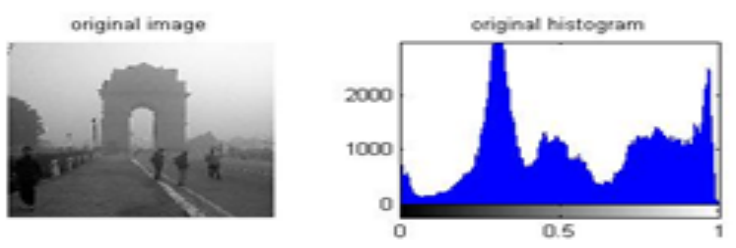

(b) Haze Image with histogram
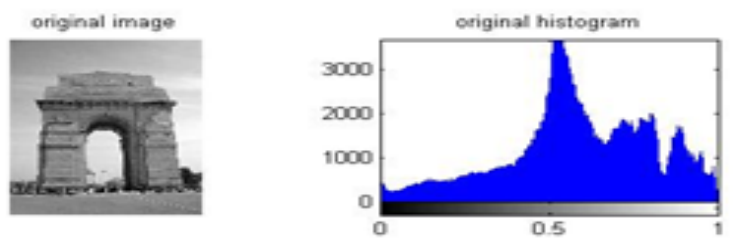

(c) Clear Image with histogram

Figure 2: different weather effect on image (a) has fog effect on image (b) Shows the haze effect

\section{NATURE INSPIRED COMPUTATIONAL INTELLIGENCE TECHNIQUES}

Many different techniques were introduced to perform different tasks on the image. Recently many new approaches are introduced which are inspired from nature. They follow the principle of Charles Darwin's "Survival of Fittest". The solutions which are best among vast pool of solutions are only forwarded to next generation or next iteration step and rest are discarded. These algorithms are broadly classified into three categories namely Modelization of human mind, Artificial Immune System and Swarm Intelligence [9].
A new wide range of nature Inspired CI based algorithm has emerged based on natural phenomenon such as behavior of social Insects in a group, migration of species in natural habitats etc. This nature Inspired intelligent technique can form the basis of building an optimization algorithm. Earlier many researcher worked effectively on digital image processing in various field like Edge detection, Color analysis, Pattern Recognition, Medical reports, Motion analysis, Restoration,Classification etc and these are the areas where effective and optimized result are obtained using nature inspired technique. The nature inspired techniques have shown better results than the other existing optimization technique for certain specific application. Also, Figure 3 below gives the proposed taxonomy of nature inspired computational intelligence techniques that can be used independently or in an integrated way for the development of optimization algorithms that can be targeted for some specific applications. A System of reality being evolutionary in nature may remain incomplete and will need revision even if the mathematically perfect reality remains in determinate. An algorithmic approach of voiding the limitations has been attempted for applications to the emerging science of mind and consciousness $[10,11]$ and the resulting technologies to prove a solution to the problem posed by classical AI Technique (The first working AI programs were written in 1951 to run on the Ferranti Mark-I machine of the University of Manchester), Computational intelligence technique based on the modelization of human mind were introduced.

\section{A. Based on the modeling of human mind}

Human brain generates thoughts and feelings which remains unknown. In the present state of human below mentioned computational intelligence technique differ from the conventional hard computing technique. All the approaches based on e form of integrated computational models of synthetic / abstract intelligence, in order to be applied to the explanation and improvement of individual and social / organizational decision-making and reasoning. The computational techniques proposed under this category are listed below. The modelization of human mind tend to be generalized to the form of integrated computational models of synthetic / abstract intelligence, in order to be applied to the explanation and improvement of individual and social / organizational decision-making and reasoning. The computational techniques proposed under this category are listed below.

- Fuzzy Set Theory

- Rough Set Theory

- Granular Computing

- Perception-Based Computing

- Wisdom Technology

- Anticipatory Computing 


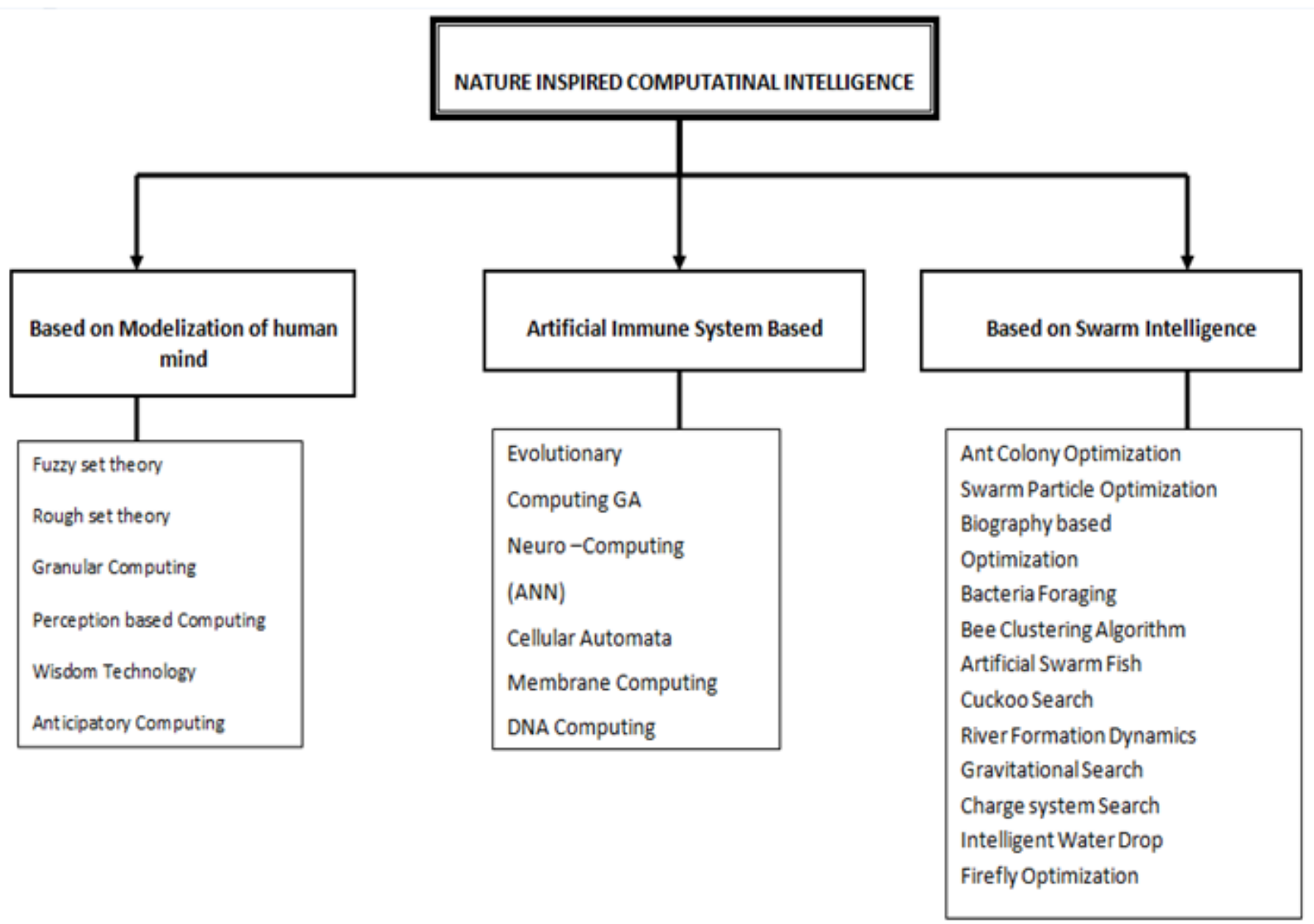

Figure 3. Taxonomy of Nature Inspired CI Techniques

\section{B. Artificial Immune based Computing}

The Immune system (IS) consists of a multitude of cells and molecules that interact in a variety of ways to detect and eliminate infectious agents (pathogens). These interactions are localized because they depend upon chemical bonding. Surfaces of immune system cells are covered with receptors, some of which are chemically bind to pathogens and some to other immune system cells or molecules to enable the complex system of signaling that mediates the immune response. Most IS cells circulate around the body via the blood and lymph systems, forming a dynamic system of distributed detection and response, where there is no centralized control Steven A. Hofmeyr et al [12]

An artificial immune system is described which incorporates many properties of natural immune systems including diversity, distributed computation, error tolerance, dynamic learning adaptation and self-monitoring. Artificial Immune System [11] is a framework to design a biologically inspired algorithm. It consists of the following basic elements:

- A representation for the components of the system

- A set of mechanisms to evaluate the interaction of individuals with the environment and each other. The environment is usually simulated by a set of input stimuli, one or more fitness function(s).

- Procedures of adaptation that govern the dynamics of the system i.e. how its behavior varies over time.
The computational intelligence techniques proposed under this category are listed below.

- Evolutionary Computing

- Neuro-computing

- Cellular Automata

- Membrane computing

\section{Swarm Intelligence Based}

The term "SWARM INTELLIGENCE" was introduced by Gerardo Beni and Jing Wang in 1989 in the context of cellular robotic systems. Bonabeau et al, 1999[13] describes swarm intelligence as the process of inter-working and intercommunication among groups of social agents to find their food. Swarm intelligence is an extension to evolutionary computation. Evolutionary computation is based on genetics whereas swarm intelligence is based on behavior of social agents like ants, bees, insects etc. The swarms work in decentralized and self organization manner. A single member of a swarm may not work properly but the group works very well.

The swarm intelligence based systems have following properties:

- There are many individuals which are homogeneous in nature.

- The individuals interact with each other using simple behavioral rules.

- The overall working of the system is affected by the interactions between individuals and environment. 
The nature inspired techniques proposed under this category are listed below.

- Ant Colony Optimization

- $\quad$ Particle Swarm Optimization

- Biogeography Based Optimization

- Bacterial Foraging Optimization

- Bee Clustering Algorithm

- Artificial Swarm fish

- Cuckoo Search

- River Formation Dynamics

- Gravitational Search

- Charged System Search

- Intelligent Water Drop

- Firefly Optimization

- Stochastic Diffusion Search

\section{NATURE INSPIRED CI TECHNIQUES FOR HARSH WEATHER}

Image Enhancement technology as one of the research works of the fuzzy image processing triggers the attention of public. Low visibility in the critical phases of the flight is one of the most disruptive factors in European aviation and causes about 50\% of arrival delays [14]. In 2007 alone at London airports more than 57000 flights were delayed because of weather. Delay cost was about 1 billion Euros per year. It is vital to increase the efficiency of air transportation system and reduce the impact of difficult weather condition.

Computational intelligence has emerged as a powerful tool for information processing, decision making, and knowledge management. The techniques of computational intelligence have been successfully developed in areas such as neural networks, fuzzy systems, and evolutionary algorithms. It is predictable that in the near future computational intelligence will play a more important role in tackling several engineering problems. Figure 4 summarizes and presents an overview of nature inspired computational intelligence and its perspective application areas along with the respective accuracy assessment metrics

\section{A. Human mind Based Approach}

> Nachiket Desai et al. [15] presents a fuzzy logic based algorithm, to de-weather fog-degraded images. Specifically, air-light estimation is carried out using fuzzy logic followed by color correction for enhanced visibility. Experimental results shown that the algorithm works effectively for images with a sky region.

$>$ Dustin fabbian and richard de dear et al. [16] presents the prediction of fog in numerical weather prediction with the help of artificial neural networks (ANNs) to provide accurate forecasts of such events at Canberra International Airport (YSCB). Results indicate that ANNs are able to offer good discrimination ability at all four lead times. The results were robust to error perturbation for various input parameters. It is recommended that such models be included when preparing forecasts for YSCB, and that the technique should be extended in its application to cover other similarly fog-prone aviation locations.

$>$ YifanWang et al. [17] presents a biologically inspired adaptive image enhancement method based on Retinex consisting of four stages: illumination estimation, reflection extraction, color restoration and post processing. The method by author is adaptive and robust to outdoor images and achieves favorable performance against state-ofthe-art methods especially for images captured under extremely hazed or low-light conditions.

\section{B. Swarm Intelligence Based}

> The research work of Adlin Sharo T et al. [18] have been carried out to enhance the degraded images using hybrid approaches such as fuzzy logic and Artificial Bee Colony (ABC) optimization technique to improve the convergence time as well as quality of the degraded images.

$>$ Ms. Dilraj Kaur, et al. [19] presented Ant Colony Optimization (ACO) approach in the problem of designing an optimal route for hard combinatorial problems by focusing on haze removal for underwater images which have poor visibility due to presence of haze. 


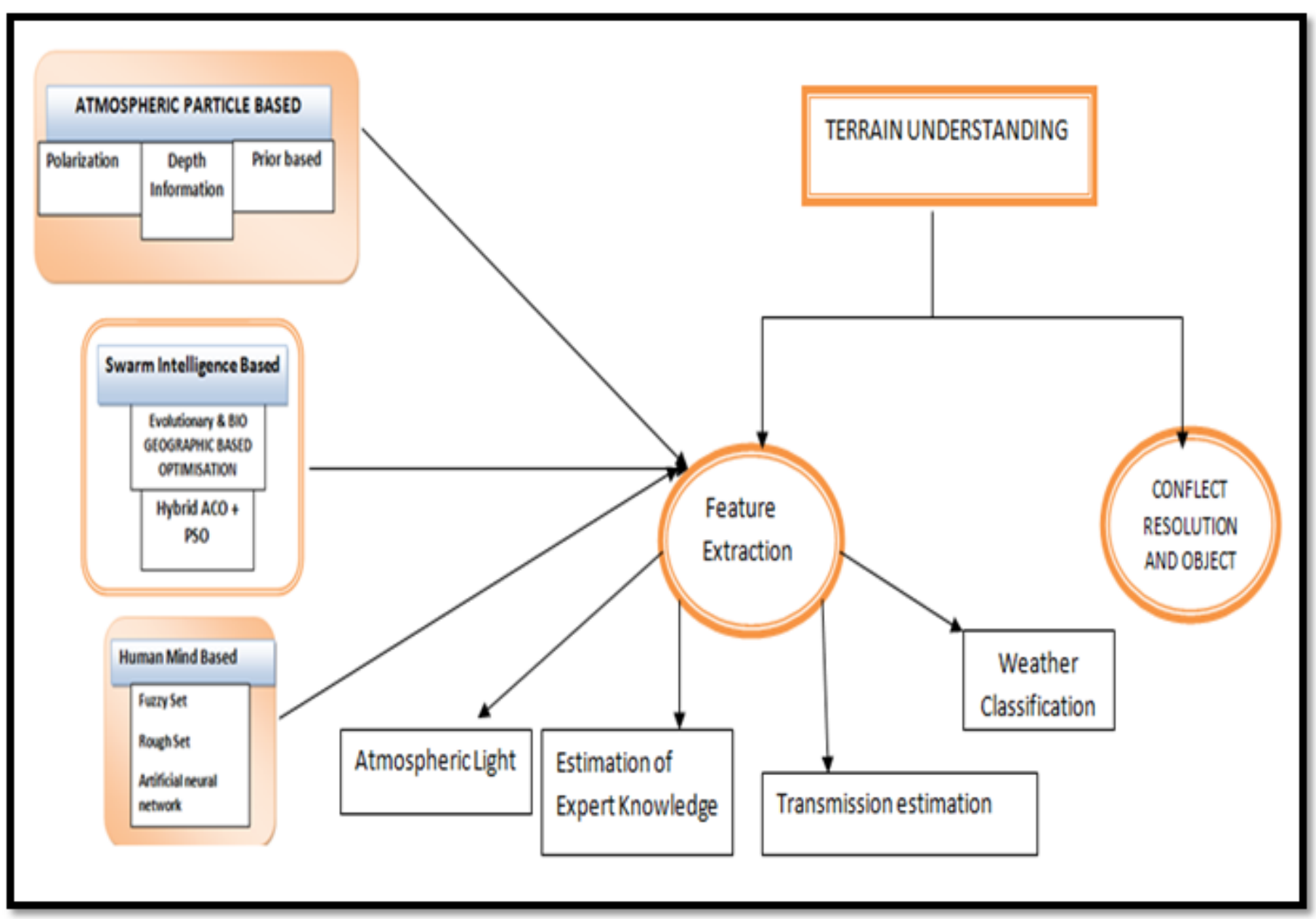

Figure 4: Overview of nature inspired computational intelligence and its application area for harsh weather

$>$ By Yuan et al. [20], stochastic particle swarm optimization (SPSO) technique's robustness is studied in regards to atmospheric aerosol size distribution estimations for a bimodal distribution that focuses on Aitken and accumulation mode aerosols. The SPSO method is used to calculate a set of 11 aerosol optical depth (AOD) values based on a size distribution and match them to an inputted set of AOD values. The AOD values for the hazy conditions lead us to suggest that testing a bimodal distribution that focuses on accumulation and course mode aerosols should be done, as the major inconsistencies in the distributions occur in these small radii ranges.

\section{CONCLUSION}

At present, it is not possible to state which technique is best for all situations as the characteristic of each image and the circumstances for each study vary so greatly. Therefore, it is essential that each analyst understand the alternative strategies for feature extraction so that he or she may be prepared to select the most appropriate technique for the feature extraction task in hand. To this end, the concept of nature inspired computational intelligence, an optimized approach for feature extraction from satellite multispectral images, has been introduced in this category. These techniques with lower cost and higher degree of classification accuracy will be able to replace high resolution high cost satellite images.
As far as feature extraction was concerned, a few nature inspired intelligent techniques like those of fuzzy sets, ant colony optimization, particle swarm optimization, biogeography based optimization etc. could be adapted and implemented for real time scenarios such as in satellite imaging applications . However, the nature inspired techniques of cuckoo Search, firefly optimization, stochastic diffusion search, geo-sciences based techniques of plate tectonics, big bang crunching etc. have not been commonly used to suit the harsh weather feature extraction applications.

\section{REFRENCES}

[1] Narasimhan, Srinivasa G., and Shree K. Nayar. "Vision and the atmosphere." International Journal of Computer Vision 48.3 (2002): 233-254.

[2] Tan, Robby T. "Visibility in bad weather from a single image." Computer Vision and Pattern Recognition, 2008. CVPR 2008. IEEE Conference on. IEEE, 2008.

[3] Zhang, Zheng, and Huadong Ma. "Multi-class weather classification on single images." Image Processing (ICIP), 2015 IEEE International Conference on. IEEE, 2015.

[4] Y.Y. Schechner, S.G. Narasimhan, and S.K. Nayar, "Instant Dehazing of Images Using Polarization,” Proc. IEEE Conf. Computer Vision and Pattern Recognition, vol. 1, pp. 325332, 2001.

[5] R. Tan, "Visibility in Bad Weather from a Single Image," Proc. IEEE Conf. Computer Vision and Pattern Recognition, June 2008. 
[6] R. Fattal, "Single Image Dehazing," Proc. ACM SIGGRAPH ’08, 2008.

[7] He, Kaiming, Jian Sun, and Xiaoou Tang. "Single image haze removal using dark channel prior." IEEE transactions on pattern analysis and machine intelligence 33.12 (2011): 2341-2353.

[8] Xu, Zhiyuan, Xiaoming Liu, and Na Ji. "Fog removal from color images using contrast limited adaptive histogram equalization." Image and Signal Processing, 2009.CISP'09.2nd International Congress on IEEE, 2009.

[9] Goel, Lavika, et al. "Taxonomy of nature inspired computational intelligence: a remote sensing perspective." Nature and Biologically Inspired Computing (NaBIC), 2012 Fourth World Congress on. IEEE, 2012.

[10] Dan Simon, "Biogeography Based Optimization", IEEE Transactions on Evolutionary Computation, Vol. 12, No. 6, 2008.

[11] Daya Gupta, Bidisha Das, and V. K. Panchal, “A Methodical Study for the Extraction of Landscape Traits Using Membrane Computing Technique”, GEM', WORLDCOMP 2011.

[12] Hofmeyr, Steven A., and Stephanie Forrest. "Architecture for an artificial immune system." Evolutionary computation 8.4 (2000): 443-473.

[13] Bonabeau, E., Dorigo, M. and Theraulaz, G.1999: Swarm intelligence. Oxford University Press
[15] Knörzer, Dietrich, and Joachim Szodruch, eds. Innovation for Sustainable Aviation in a Global Environment: Proceedings of the Sixth European Aeronautics Days. IOS Press, 2012.

[16] Desai, Nachiket, et al. "A fuzzy logic based approach to deweather fog-degraded images." Computer Graphics, Imaging and Visualization, 2009. CGIV'09. Sixth International Conference on. IEEE, 2009.

[17] Fabbian, Dustin, Richard de Dear, and Stephen Lellyett. "Application of artificial neural network forecasts to predict fog at Canberra International Airport." Weather and forecasting 22.2 (2007): 372-381.

[18] Wang, Yifan, et al. "Biologically inspired image enhancement based on Retinex." Neurocomputing 177 (2016): 373-384.

[19] Adlin Sharo, T., and Kumudha Raimond. "Enhancing degraded color images using Fuzzy logic and artificial Bee colony." International Journal Of Computational Engineering Research (ijceronline. com) 3.3 (2013).

[20] Kaur, Ms Dilraj, and Ms Pooja. "EXTENDED RESULTS: ACO BASED MIX-CLAHE FOR UNDERWATER HAZE REMOVAL."

[21] Yuan, Yuan, et al. "Inverse problem for particle size distributions of atmospheric aerosols using stochastic particle swarm optimization." Journal of Quantitative Spectroscopy and Radiative Transfer 111.14 (2010): 21062114. 